Tôhoku Math. Journ.

28 (1976), 497-509.

\title{
AN APPROXIMATE HILBERT TRANSFORM AND ITS INVERSION
}

\section{J. N. Pandey AND Edward Hughes}

(Received August 12, 1974)

\footnotetext{
Abstract. If $\eta>0$ and $f$ is in a certain space of generalized functions, define

$$
F_{\eta}(x)=\left\langle f(t), \frac{t-x}{(t-x)^{2}+\eta^{2}}\right\rangle \text {. }
$$

It is shown that
}

$$
\lim _{\eta \rightarrow 0+}\left(-\frac{1}{\pi^{2}}\right) P \int_{-\infty}^{\infty} \frac{F_{\eta}(x) d x}{x-y}=f(y)
$$

in the weak distributional sense.

Introduction. If $f$ is a function in $L^{\infty}(-\infty, \infty)$, then its Hilbert transform is defined to be

$$
H f(x)=P \int_{-\infty}^{\infty} \frac{f(t)}{t-x} d t,
$$

where the " $P$ " denotes a principal-value integral. The inversion formula is

$$
-\frac{1}{\pi^{2}} H(H(f))=f,
$$

and it can be shown [9, Chap. 5] that

$$
H f(x)=\lim _{\eta \rightarrow 0+} \int_{-\infty}^{\infty} \frac{f(t)(t-x) d t}{(t-x)^{2}+\eta^{2}},
$$

the limit being in the topology of $L^{p}(-\infty, \infty)$, from which it follows that

$$
\lim _{\eta \rightarrow 0+}-\frac{1}{\pi^{2}} H\left(\int_{-\infty}^{\infty} \frac{(t-x) f(t) d t}{(t-x)^{2}+\eta^{2}}\right)=f .
$$

In this paper we shall explore the possibilities of extending these results to certain spaces of generalized functions. It will be shown that the "approximate" inversion formula (2) holds for distributions, but the A8747.

This work was supported by National Research Council of Canada grants Nos. A5298 and 
classical inversion formula (1) involves some subtle questions of interpretation. There is some overlap with the results of [2] and [4]; however, our results are not restricted to distributions of compact support, and are more constructive than those of [4].

The notation and terminology follows that of [11]. The set of real numbers is denoted by $R$, and $t, x$ and $y$ are real variables unless otherwise stated. If $f$ is a generalized function, then the notation $f(t)$ is used to indicate that the testing functions on which $f$ is defined have $t$ as their variable. The pairing between a testing-function space and its dual is denoted by $\langle f, \phi\rangle$. The space of $C^{\infty}$ functions on $R$ having compact support is denoted by $\mathscr{D}$. Its dual is the space of Schwartz distributions on $R$. [8, Vol. I, p. 65]. We use the terms "distribution" and "generalized function" interchangeably.

I. The testing function space $H_{\alpha}$. Let $\alpha$ be a fixed real number satisfying $0<\alpha<1$. A complex-valued $C^{\infty}$-function $\phi$ on $(-\infty, \infty)$ is said to belong to $H$ if for each $k=0,1,2, \cdots, \gamma_{k}(\phi)=\sup _{t \in R}\left|\xi(t) \phi^{(k)}(t)\right|<\infty$, where $\xi(t)$ is a positive $C^{\infty}$ function satisfying

$$
\xi(t)= \begin{cases}1, & |t| \leqq 1 \\ |t|^{\alpha-1}, & |t| \geqq 2 .\end{cases}
$$

The topology of $H_{\alpha}$ is generated by the seminorms $\left\{\gamma_{k}\right\}$ in the usual manner [9; p. 8]. In particular, a sequence $\left\{\phi_{n}\right\}$ converges to a function $\phi$ in the topology of $H_{\alpha}$ if and only if $\xi(t) \phi_{n}^{(k)}(t) \rightarrow \xi(t) \phi^{(k)}(t)$ as $n \rightarrow \infty$, uniformly in $t$, for all $k=0,1,2, \cdots$, . It can be shown that $H_{\alpha}$ is a locally convex, sequentially complete Hausdorff topological vector space, closed under differentiation. It is easy to check that for fixed $\eta>0$ and complex $y$ with $\operatorname{Im} y \neq \pm \eta$, the function $(y-t) /\left((y-t)^{2}+\eta^{2}\right)$ belongs to $H_{\alpha}$. If $f$ is in the distribution space $H_{\alpha}^{\prime}$, we define a function $F_{\eta}(y)=$ $\left\langle f(t),(y-t) /\left((y-t)^{2}+\eta^{2}\right)\right\rangle$, which may be thought of as an approximation to the Hilbert transform.

THEOREM 1. Let $\eta>0$, let $\Omega$ be the complex domain $\{\operatorname{Im} z \neq \pm \eta\}$, and let $y \in \Omega$. Then $F_{\eta}(y)$ as defined above is analytic in $\Omega$, and

$$
F_{\eta}^{(k)}(y)=\left\langle f(t), \frac{\partial^{k}}{\partial y^{k}}\left(\frac{y-t}{(y-t)^{2}+\eta^{2}}\right)\right\rangle .
$$

In addition, if $y$ is considered a real variable, then $F_{\eta}(y)$, as a function of $y$ and $\eta$, is harmonic in the upper half-plane.

The theorem may be proved by a technique similar to that of [5, Theorem 1]. 
The main result of this paper is the following theorem, which will be proved in Section III.

TheOREM 2. For fixed $\alpha \in(0,1), \eta>0$, and $f \in H_{\alpha}^{\prime}$, we have

$$
\lim _{\eta \rightarrow 0+} \lim _{N \rightarrow \infty}-\frac{1}{\pi^{2}} P \int_{-N}^{N} \frac{F_{\eta}(x) d x}{x-y}=f(y)
$$

in the weak distributional sense.

II. Some lemmas. The following results are preliminaries to the proof of the inversion formula, Theorem 2.

LemMA 1. For fixed real $y, \eta>0$, define

$$
\psi_{\varepsilon}(t)=\frac{t-y}{(t-y)^{2}+\eta^{2}} \ln \frac{(y-t-\varepsilon)^{2}+\eta^{2}}{(y-t+\varepsilon)^{2}+\eta^{2}} .
$$

Then as $\varepsilon \rightarrow 0+, \psi_{\varepsilon}(t) \rightarrow 0$ in the topology of $H_{\alpha}$.

Proof. One can check that

$$
\ln \left|\frac{(y-t-\varepsilon)^{2}+\eta^{2}}{(y-t+\varepsilon)^{2}+\eta^{2}}\right| \rightarrow 0 \text { as } \varepsilon \rightarrow 0+, \text { uniformly in } t .
$$

Hence $\xi(t) \psi_{\varepsilon}(t) \rightarrow 0$ as $\varepsilon \rightarrow 0+$, uniformly in $t$. By an inductive argument whose details we omit one now shows that $\xi(t) \psi_{\varepsilon}^{(k)}(t) \rightarrow 0$ as $\varepsilon \rightarrow 0+$, uniformly in $t$, for each $k=0,1,2, \cdots$, which proves the lemma. For details of the proof, see [7].

LEMMA 2. For fixed $\eta>0$ and real $x$, the function

$$
\tan ^{-1}\left(\frac{t-x+\varepsilon}{\eta}\right)-\tan ^{-1}\left(\frac{t-x-\varepsilon}{\eta}\right) \rightarrow 0 \text { in } H_{\alpha} \text { as } \varepsilon \rightarrow 0+.
$$

The proof is straightforward and will be omitted.

LEMMA 3. Let $\phi$ be a $C^{\infty}$-function on $R$ with compact support contained in $(a, b)$, and let $\eta>0$ be fixed. Then

$$
\lim _{N \rightarrow \infty} \ln \left(\frac{(N-t)^{2}+\eta^{2}}{(N+t)^{2}+\eta^{2}}\right) \int_{a}^{b} \frac{(t-y) \phi(y) d y}{(t-y)^{2}+\eta^{2}}=0 \text { in the topology of } H_{\alpha} \text {. }
$$

Proof. We write

$$
f_{N}(t)=\ln \left(\frac{(N-t)^{2}+\eta^{2}}{(N+t)^{2}+\eta^{2}}\right) ; \quad g(t)=\int_{a}^{b} \frac{(t-y) \phi(y) d y}{(t-y)^{2}+\eta^{2}}
$$

and

$$
r_{y}(t)=\frac{t-y}{(t-y)^{2}+\eta^{2}}
$$


We want to show that $f_{N}(t) g(t) \rightarrow 0$ in $H_{\alpha}$ as $N \rightarrow \infty$, which means

$$
\begin{aligned}
\xi(t) D_{t}^{k}\left(f_{N}(t) g(t)\right) \rightarrow 0 \quad \text { as } \quad N \rightarrow \infty, \text { uniformly in } t, & \text { for each } k=0,1,2, \cdots .
\end{aligned}
$$

First we take $k=0$. A lengthy but routine computation shows that if $|t| \leqq \sqrt{N}$, then

$$
\ln \frac{(N-t)^{2}+\eta^{2}}{(N+t)^{2}+\eta^{2}} \leqq \frac{(N+\sqrt{N})^{2}+\eta^{2}}{(N-\sqrt{\bar{N}})^{2}+\eta^{2}} \cdot \frac{4 N^{3 / 2}}{(N-\sqrt{N})^{2}+\eta^{2}} \rightarrow 0 \text { as } N \rightarrow \infty .
$$

If, on the other hand, $|t|>\sqrt{N}$, we note that if $t \geqq \max (|a|,|b|)$, then $\left|r_{y}(t)\right|$ is monotone decreasing in $t$, and hence, under the assumption $|t| \geqq \sqrt{N}$, we have $\left|r_{y}(t)\right| \leqq(\sqrt{N}-y) /\left((\sqrt{N}-y)^{2}+\eta^{2}\right)$, if $N$ is sufficiently large. Now we assume $t \geqq 0$; a similar argument holds if $t$ is negative; we have $\left((N-t)^{2}+\eta^{2}\right) /\left((N+t)^{2}+\eta^{2}\right) \leqq 1$; and the minimum of this function is at $t=\sqrt{N^{2}+\eta^{2}}$; so that

$$
\frac{\left(N-\sqrt{N^{2}+\eta^{2}}\right)^{2}+\eta^{2}}{\left(N+\sqrt{N^{2}+\eta^{2}}\right)^{2}+\eta^{2}} \leqq \frac{(N-t)^{2}+\eta^{2}}{(N+t)^{2}+\eta^{2}} \leqq 1, \quad \text { for all } t \geqq 0 \text {. }
$$

From this it follows that

$$
\left|\ln \frac{(N-t)^{2}+\eta^{2}}{(N+t)^{2}+\eta^{2}}\right| \leqq \ln \frac{\left(N+\sqrt{N^{2}+\eta^{2}}\right)^{2}+\eta^{2}}{\left(N-\sqrt{N^{2}+\eta^{2}}\right)^{2}+\eta^{2}}=P_{N},
$$

which is bounded in $N$. So for $N$ sufficiently large, and $t \geqq \sqrt{N}$, we have

$$
\left|\xi(t) f_{N}(t) r_{y}(t)\right| \leqq C P_{N} \frac{\sqrt{\bar{N}}-y}{(\sqrt{\bar{N}}-y)^{2}+\eta^{2}},
$$

where $C$ is a constant. The right-hand side has the limit zero, uniformly in $y \in[a, b]$.

Putting the above together, we find that $\xi(t) f_{N}(t) g(t) \rightarrow 0$ uniformly in $t$, as $N \rightarrow \infty$. We now consider $k=1$ : let $K_{N}(t)=2(N+t) /\left((N+t)^{2}+\right.$ $\left.\eta^{2}\right)$. Then a simple computation shows

$$
\begin{aligned}
\frac{d}{d t} f_{N}(t) r_{y}(t)= & f_{N}(t) \frac{\eta^{2}-(t-y)^{2}}{\left((t-y)^{2}+\eta^{2}\right)^{2}} \\
& +K_{N}(t) r_{y}(t)+K_{N}(-t) r_{y}(t) .
\end{aligned}
$$

Now $\left(r_{y}(t)\right) \leqq 1 / 2 \eta$, and so for $|t| \leqq \sqrt{N}$,

$$
\left|\xi(t) K_{N}(t) r_{y}(t)\right| \leqq \frac{C(N+\sqrt{N})}{(N-\sqrt{N})^{2}+\eta^{2}} \cdot \frac{1}{2 \eta} \rightarrow 0 \quad \text { as } \quad N \rightarrow \infty,
$$


and for $|t| \geqq \sqrt{N}$,

$$
\begin{aligned}
&\left|\xi(t) K_{N}(t) r_{y}(t)\right| \leqq \frac{C}{\eta} \frac{\sqrt{N}-y}{(\sqrt{\bar{N}}-y)^{2}+\eta^{2}} \rightarrow 0, \\
& \text { uniformly in } y \in a, b .
\end{aligned}
$$

Similarly one shows that

$$
\xi(t) K_{N}(-t) r_{y}(t) \rightarrow 0 \text { as } N \rightarrow \infty \text {, uniformly in } t \geqq 0 .
$$

Now we see that $\xi(t)\left|r_{y}^{\prime}(t)\right| \leqq 2 C / \eta^{2}$ for all $y$ and $t$; by considering separately the cases $t \leqq \sqrt{N}$ and $t \geqq \sqrt{N}$, as above, we can show that

$$
\xi(t) f_{N}(t) r_{y}^{\prime}(t) \rightarrow 0 \text { uniformly in } t \text { and } y \in[a, b] \text {. }
$$

From (7), (8), (9) and (10) we now have $\xi(t) D_{t}\left(f_{N}(t) r_{y}(t)\right) \rightarrow 0$ as $N \rightarrow \infty$ uniformly in $t$ and in $y \in[a, b]$. This is the case $k=1$. We now must treat the case $k>1$, which we do by induction. For any $m \geqq 1$, the Leibnitz formula yields

$$
D^{m}\left(f_{N}(t) r_{y}(t)\right)=f_{N}(t) r_{y}^{(m)}(t)+\sum_{i=1}^{m}\left[K_{N}^{(i-1)}(t)+K_{N}^{(i-1)}(-t)\right] r_{y}^{(m-i)}(t) .
$$

And so it suffices to show that

$$
\begin{aligned}
& \text { (i) } \xi(t) f_{N}(t) r_{y}^{(k)}(t) \rightarrow 0 \\
& \text { (ii) } \xi(t) K_{N}^{(i-1)}( \pm t) r_{y}^{(k-i)}(t) \rightarrow 0
\end{aligned}
$$

as $N \rightarrow \infty$ uniformly in $t$ and in $y \in(a, b)$, for all $k \geqq 1$ and $i=1, \cdots, k$. Assuming now that (11) holds for all $k \leqq n$, we wish to show that it holds as well for $k=n+1$. We note that, for $j \geqq 1$,

$$
\begin{aligned}
r_{y}^{(j+1)}(t) & =\frac{-2\left(\begin{array}{c}
j+1 \\
1
\end{array}\right)(t-y) r_{y}^{(j)}(t)+\left(\begin{array}{c}
j+1 \\
2
\end{array}\right) r_{y}^{(j-1)}(t)}{(t-y)^{2}+\eta^{2}} \\
& =q_{1}(t) r_{y}^{(j)}(t)+q_{2}(t) r_{y}^{(j-1)}(t),
\end{aligned}
$$

where $q_{1}$ and $q_{2}$ are both bounded functions of $t$.

Now (11) (i) becomes

$$
\begin{aligned}
\xi(t) f_{N}(t) r_{y}^{(n+1)}(t)= & \xi(t) q_{1}(t) f_{N}(t) r_{y}^{(n)}(t) \\
& +\xi(t) q_{2}(t) f_{N}(t) r_{y}^{(n-1)}(t) \rightarrow 0
\end{aligned}
$$

uniformly in $t, y$ as $N \rightarrow \infty$, by the induction hypothesis.

To prove (11) (ii), we first note

$$
\begin{aligned}
\xi(t) K_{N}^{(i-1)}(t) r_{y}^{(n+1-i)}(t)= & q_{1}(t) \xi(t) K_{N}^{(i-1)}(t) r_{y}^{(n-i)}(t) \\
& +q_{2}(t) \xi(t) K_{N}^{(i-1)}(t) r_{y}^{(n-i-1)}(t) .
\end{aligned}
$$


If $1 \leqq i \leqq n-1$, then the induction hypothesis applies directly to the right side of (13). If $i=n$, we have

$$
\xi(t) K_{N}^{(n-1)}(t) r_{y}^{\prime}(t)=\xi(t) K_{N}^{(n-1)}(t) \frac{\left(1-2(t-y) r_{y}(t)\right)}{(t-y)^{2}+\eta^{2}}
$$

which is easily shown to go to zero as $N \rightarrow \infty$, uniformly in $t$ and in $y \in[a, b]$.

If $i=n+1$, then $K^{(n)}(t)=q_{2}(t) K^{(n-1)}(t)+q_{2}(t) K^{(n-2)}(t)$; where $q_{1}$ and $q_{2}$ are bounded functions, and so

$$
\begin{aligned}
\xi(t) K^{(n)}(t) r_{y}(t)= & q_{1}(t) \xi(t) K^{(n-1)}(t) r_{y}(t)+q_{2}(t) \xi(t) K^{(n-2)}(t) r_{y}(t) \\
& \rightarrow 0 \text { as } N \rightarrow \infty
\end{aligned}
$$

uniformly in $t$, and in $y \in[a, b]$, by the induction hypothesis. This completes the proof of Lemma 3 .

LemMA 4. If $\phi$ is as in Lemma 3, then

$$
\lim _{N \rightarrow \infty} \int_{-\infty}^{\infty} \frac{(t-y)}{(t-y)^{2}+\eta^{2}} \ln \left|\frac{N-y}{N+y}\right| \phi(y) d y=0 \text { in the topology of } H_{\alpha} \text {. }
$$

Proof. If $N>b$ and $k \geqq 0$, we have

$$
\begin{aligned}
\xi(t)\left(\frac{d}{d t}\right)^{k} & \int_{-\infty}^{\infty} \frac{t-y}{(t-y)^{2}+\eta^{2}} \ln \left|\frac{N-y}{N+y}\right| \phi(y) d y \\
& =\xi(t) \int_{a}^{b}\left(-\frac{d}{d y}\right)^{k} \frac{t-y}{(t-y)^{2}+\eta^{2}} \ln \left|\frac{N-y}{N+y}\right| \phi(y) d y \\
& =\xi(t) \int_{a}^{b} \frac{t-y}{(t-y)^{2}+\eta^{2}}\left[\left(\frac{d}{d y}\right)^{k}\left(\ln \left|\frac{N-y}{N+y}\right| \phi(y)\right)\right] d y .
\end{aligned}
$$

The absolute value of the expression in (15) is bounded by

$$
\frac{C}{2 \eta} \int_{a}^{b}\left|\left(\frac{d}{d y}\right)^{k}\left[\ln \left|\frac{N-y}{N+y}\right| \phi(y)\right]\right| d y \rightarrow 0 \text { as } N \rightarrow \infty \text {. }
$$

LEMMA 5. Let $\phi, \eta$ be as in Lemma 3, and let $k \geqq 1$ be a positive integer. Then

$$
\begin{aligned}
\xi(t) \frac{1}{\pi^{2}} D_{t}^{k} & {\left[\tan ^{-1}\left(\frac{N+t}{\eta}\right)+\tan ^{-1}\left(\frac{N-t}{\eta}\right)\right] } \\
\times & {\left[\int_{-\infty}^{\infty} \frac{\eta}{(y-t)^{2}+\eta^{2}} \phi(y) d y\right] \rightarrow 0 }
\end{aligned}
$$

as $N \rightarrow \infty$, uniformly in $t$.

Proof. A simple computation shows that 


$$
\begin{gathered}
\left|D_{t}^{k}\left(\tan ^{-1}\left(\frac{N+t}{\eta}\right)\right)\right| \leqq \frac{2(k-1) !}{\eta^{k}} \quad \text { for all } t \text {, and all } \\
k=1,2,3, \cdots .
\end{gathered}
$$

Denote the expression in (16) by $I$. For each $k$ we have

$$
|I| \leqq \frac{2(k-1) !}{\eta^{k} \pi^{2}} \int_{-\infty}^{\infty} \frac{\eta}{(y-t)^{2}+\eta^{2}}|\phi(y)| d y .
$$

For an arbitrary $\varepsilon>0$, we can find $M>0$ such that

$$
|I| \leqq \varepsilon \text { for }|t| \geqq M \text {. }
$$

It is easy to check that for each $k$,

$$
D_{t}^{k}\left[\tan ^{-1}\left(\frac{N+t}{\eta}\right)+\tan ^{-1}\left(\frac{N-t}{\eta}\right)\right] \rightarrow 0 \quad \text { as } \quad N \rightarrow \infty,
$$

uniformly on any compact set of real numbers. The integral $\int_{-\infty}^{\infty}\left(\eta /\left((y-t)^{2}+\eta^{2}\right)\right)|\phi(y)| d y$ can be bounded by a constant independent of $t$, and thus having chosen $M$ as above, we can choose $N$ large enough to insure that $|I|<\varepsilon$ for all $t$.

Lemma 6. For fixed $\eta>0$, real $t$, and $\phi(y) \in \mathscr{D}$,

$$
\begin{gathered}
\lim _{\eta \rightarrow 0+} \lim _{N \rightarrow \infty} \frac{1}{\pi^{2}}\left[\tan ^{-1}\left(\frac{N+t}{\eta}\right)+\tan ^{-1}\left(\frac{N-t}{\eta}\right)\right] \\
\quad \times \int_{-\infty}^{\infty} \frac{\eta}{(y-t)^{2}+\eta^{2}} \phi(y) d y=\phi(t),
\end{gathered}
$$

in the topology of $H_{\alpha}$.

Proof. A simple computation shows that

$$
\begin{aligned}
& \xi(t) D_{t}^{k}\left[\left[\tan ^{-1}\left(\frac{N+t}{\eta}\right)+\tan ^{-1}\left(\frac{N-t}{\eta}\right)\right] \int_{-\infty}^{\infty} \frac{\eta}{(y-t)^{2}+\eta^{2}} \phi(y) d y\right] \\
& =\xi(t)\left[\tan ^{-1}\left(\frac{N+t}{\eta}\right)+\tan ^{-1}\left(\frac{N-t}{\eta}\right)\right] \int_{-\infty}^{\infty} \frac{\eta}{(y-t)^{2}+\eta^{2}} \phi^{(k)}(y) d y \\
& \quad+\xi(t) \sum_{j=1}^{k}\left(\begin{array}{l}
k \\
j
\end{array}\right) D_{t}^{k}\left[\tan ^{-1}\left(\frac{N+t}{\eta}\right)+\tan ^{-1}\left(\frac{N-t}{\eta}\right)\right] \\
& \quad \times \int_{-\infty}^{\infty} \frac{\eta}{(y-t)^{2}+\eta^{2}} \phi^{(k-j)}(y) d y .
\end{aligned}
$$

From Lemma 5 it follows that the expression in (19) has the limit

$$
\xi(t)\left[\tan ^{-1}\left(\frac{N+t}{\eta}\right)+\tan ^{-1}\left(\frac{N-t}{\eta}\right)\right] \int_{-\infty}^{\infty} \frac{\eta}{(y-t)^{2}+\eta^{2}} \phi^{(k)}(y) d y
$$


uniformly in $t$ as $N \rightarrow \infty$.

The proof therefore reduces to showing that

$$
\begin{aligned}
& \xi(t)\left[\tan ^{-1}\left(\frac{N+t}{\eta}\right)+\tan ^{-1}\left(\frac{N-t}{\eta}\right)\right] \\
& \quad \times \int_{-\infty}^{\infty} \frac{\eta}{(y-t)^{2}+\eta^{2}}\left[\phi^{(k)}(y)-\phi^{(k)}(t)\right] d y \rightarrow 0
\end{aligned}
$$

uniformly in $t$, as $N \rightarrow \infty$ and $\eta \rightarrow 0+$ in succession. Let $\delta \in(0,1)$ and divide the range of integration in (20) into subintervals $(-\infty, t-\delta)$, $(t-\delta, t+\delta)$, and $(t+\delta, \infty)$; denote the corresponding expressions in (20) by $I_{1}, I_{2}$, and $I_{3}$, respectively. Then since $\xi(t)$ and $\tan ^{-1}((N+t) / \eta)$ are bounded independently of $t, N$, and $\eta$, there is a constant $C$ such that

$$
\begin{aligned}
\left|I_{2}\right| & \leqq C \delta M \int_{t-\delta}^{t+\delta} \frac{\eta}{(y-t)^{2}+\eta^{2}} d y, \quad\left(\text { where } M=\sup _{-\infty<x<\infty}\left|\phi^{(k+1)}(x)\right|\right) \\
& \leqq C \delta M \pi .
\end{aligned}
$$

Given $\varepsilon>0$, choose $\delta$ so that $C M \pi \delta<\varepsilon$. Now

$$
\begin{aligned}
\left|I_{3}\right| & \leqq 2 C P \pi \int_{t+\delta}^{\infty} \frac{\eta}{(y-t)^{2}+\eta^{2}} d y ; \quad\left(\text { where } P=\sup \left|\phi^{k}(x)\right|\right) \\
& =2 C P \pi\left[\frac{\pi}{2}-\tan ^{-1}\left(\frac{\delta}{\eta}\right)\right] \rightarrow 0 \quad \text { as } \quad \eta \rightarrow 0+.
\end{aligned}
$$

So we find that

$$
\lim _{\eta \rightarrow 0+} \lim _{N \rightarrow \infty} I_{3}=0 \text { uniformly in } t \text {. }
$$

The same argument holds for $I_{1}$, so it follows that

$$
\lim _{\eta \rightarrow 0+} \lim _{N \rightarrow \infty}\left(I_{1}+I_{2}+I_{3}\right)=0 \text {, uniformly in } t \text {. }
$$

This completes the proof of Lemma 6 .

LEMMA 7. If $f \in H_{\alpha}^{\prime}$, a efine $F_{\eta}(x)=\left\langle f(t),(t-x) /\left((t-x)^{2}+\eta^{2}\right)\right\rangle$. Then for $y$ real and $N>0$.

where

$$
\begin{aligned}
P \int_{-N}^{N} F_{\eta}(x) \frac{1}{x-y} d x & =\left\langle f(t), P \int_{-N}^{N} \frac{t-x}{(t-x)^{2}+\eta^{2}} \frac{1}{x-y} d x\right\rangle \\
& =\left\langle f(t), G_{N}(t, y, \eta)\right\rangle
\end{aligned}
$$

$$
\begin{aligned}
G_{N}(t, y, \eta)= & \frac{t-y}{(t-y)^{2}+\eta^{2}} \ln \left|\frac{N-y}{N+y}\right| \\
& -\frac{\eta}{(t-y)^{2}+\eta^{2}}\left[\tan ^{-1}\left(\frac{N+t}{\eta}\right)+\tan ^{-1}\left(\frac{N-t}{\eta}\right)\right]
\end{aligned}
$$




$$
-\frac{t-y}{2(t-y)^{2}+\eta^{2}} \ln \frac{(N-t)^{2}+\eta^{2}}{(N+t)^{2}+\eta^{2}}
$$

Proof. Let $\varepsilon>0$. We note

$$
P \int_{-N}^{N} F_{\eta}(x) \frac{1}{x-y} d x=\lim _{\varepsilon \rightarrow 0+}\left(\int_{-N}^{y-\varepsilon}+\int_{y+\varepsilon}^{N}\right) \frac{F_{\eta}(x)}{x-y} d x
$$

By a Riemann-Sum argument similar to [6, Th. 2], one can show that

$$
\left(\int_{-N}^{y-\varepsilon}+\int_{y+\varepsilon}^{N}\right) \frac{F_{\eta}(x)}{x-y} d x=\left\langle f(t), G_{N}(t, y)-K_{N}(t, y, \varepsilon)\right\rangle,
$$

where

$$
\begin{aligned}
K_{N}(t, y, \varepsilon)= & \frac{\eta}{\eta^{2}+(t-y)^{2}}\left[\tan ^{-1}\left(\frac{y-t-\varepsilon}{\eta}\right)-\tan ^{-1}\left(\frac{y+\varepsilon-t}{\eta}\right)\right] \\
& +\frac{t-y}{2\left((t-y)^{2}+\eta^{2}\right)} \ln \frac{(y-t-\varepsilon)^{2}+\eta^{2}}{(y-t+\varepsilon)^{2}+\eta^{2}} .
\end{aligned}
$$

It follows from Lemmas 1 and 2 that $K_{N}(t, y, \varepsilon) \rightarrow 0$ in $H_{\alpha}$ as $\varepsilon \rightarrow 0+$. Therefore taking limits as $\varepsilon \rightarrow 0+$ in (21), we have

$$
P \int_{-N}^{N} \frac{F_{\eta}(x)}{x-y} d x=\left\langle f(t), G_{N}(t, y, \eta)\right\rangle .
$$

III. The inversion formula. We now prove Theorem 2:

Let $\phi \in \mathscr{D}$, with the support of $\phi$ in $(a, b)$. Then

$$
\begin{aligned}
\left\langle P \int_{-N}^{N}\right. & \left.\left\langle f(t), \frac{t-x}{(t-x)^{2}+\eta^{2}}\right\rangle \frac{1}{x-y} d x, \phi(y)\right\rangle \\
& =\left\langle\left\langle f(t), G_{N}(t, y, \eta)\right\rangle, \phi(y)\right\rangle \text { by Lemma } 7, \\
& =\left\langle f(t), \int_{a}^{b} G_{N}(t, y, \eta) \phi(y) d y\right\rangle, \quad \text { by a Riemann-Sum argument }
\end{aligned}
$$

as in [6, Th. 2].

Now Lemmas 2, 3 and 4 give

$$
\begin{aligned}
& \lim _{\eta \rightarrow 0+} \lim _{N \rightarrow \infty}\left\langle-\frac{1}{\pi^{2}} P \int_{-N}^{N} \frac{F_{\eta}(x)}{x-y} d x, \phi(y)\right\rangle \\
& =\lim _{\eta \rightarrow 0+} \lim _{N \rightarrow \infty}\left\langle f(t), \frac{1}{\pi^{2}}\left[\tan ^{-1}\left(\frac{N+t}{\eta}\right)+\tan ^{-1}\left(\frac{N-t}{\eta}\right)\right] \int_{a}^{b} \frac{\eta \phi(y) d y}{(t-y)^{2}+\eta^{2}}\right\rangle \\
& =\langle f(t), \phi(t)\rangle, \text { by Lemma } 6 .
\end{aligned}
$$

This completes the proof.

The following uniqueness result is an immediate consequence of Theorem 2:

THEOREM 3. If $f, g \in H_{\alpha}^{\prime}$, and $F_{\eta}(y)=G_{\eta}(y)$ for all real $y$, and all 
$\eta>0$, then $f=g$ in the sense of equality over $\mathscr{D}$.

It is not difficult to show [10, p. 101-102] that the closure of the space $\mathscr{D}$ in $H_{\alpha}$ is the subspace $\underline{H}_{\alpha}$ consisting of those functions $\phi \in H_{\alpha}$ such that

$$
\lim _{x \rightarrow \pm \infty} \phi^{(k)}(x)=0 \text { for all } k=0,1,2, \cdots \text {. }
$$

It follows that Theorem 3 holds if $\mathscr{D}$ is replaced by $\underline{H}_{\alpha}$. One may not replace $\mathscr{D}$ by $H_{\alpha}$ in Theorem 3 , as the counter example of a "Banach limit" at $+\infty$ shows; indeed $\underline{H}_{\alpha}$ is the largest testing function space over which the uniqueness result holds.

Theorem 2 shows that

$$
\lim _{\eta \rightarrow 0+} \lim _{N \rightarrow \infty}\left\langle-\frac{1}{\pi^{2}} P \int_{-N}^{N} \frac{F_{\eta}(x)}{x-y} d x, \phi(y)\right\rangle=\langle f, \phi\rangle .
$$

We can also move the inner limit inside the brackets and obtain

THEOREM 4. Under the hypotheses of Theorem 3,

(23) $\lim _{\eta \rightarrow 0+} P \int_{-\infty}^{\infty} \frac{F_{\eta}(x) d x}{x-y}=-\pi^{2} f(y)$, in the weak distributional sense.

Proof. (Outline): We have

$$
P \int_{-N}^{N} \frac{F_{\eta}(x)}{x-y} d x=\left\langle f(t), P \int_{-N}^{N} \frac{t-x}{(t-x)^{2}+\eta^{2}} \cdot \frac{1}{x-y} d x\right\rangle
$$

(by an argument similar to [6, Th. 2])

$$
\begin{aligned}
= & \left\langle f(t), \frac{t-y}{(t-y)^{2}+\eta^{2}} \ln \left|\frac{N-y}{N+y}\right|+\frac{1}{2} \frac{t-y}{(t-y)^{2}+\eta^{2}} \ln \frac{(N-t)^{2}+\eta^{2}}{(N+t)^{2}+\eta^{2}}\right. \\
& \left.-\frac{\eta\left[\tan ^{-1}\left(\frac{N-t}{\eta}\right)+\tan ^{-1}\left(\frac{N+t}{\eta}\right)\right]}{(t-y)^{2}+\eta^{2}}\right\rangle \rightarrow\left\langle f(t), \frac{-\eta \pi}{(t-y)^{2}+\eta^{2}}\right\rangle
\end{aligned}
$$

as $N \rightarrow \infty$, by arguments similar to previous lemmas. So we have

$$
-\frac{1}{\pi^{2}} P \int_{-\infty}^{\infty} \frac{F_{\eta}(x)}{(x-y)} d x=\left\langle f(t), \frac{1}{\pi} \frac{\eta}{(t-y)^{2}+\eta^{2}}\right\rangle \text {. }
$$

If $\phi \in \mathscr{D}$, then

$$
\left\langle-\frac{1}{\pi^{2}} P \int_{-\infty}^{\infty} \frac{F_{\eta}(x) d x}{x-y}, \phi(y)\right\rangle=\left\langle f(t), \frac{1}{\pi} \int_{-\infty}^{\infty} \frac{\eta}{(t-y)^{2}+\eta^{2}} \phi(y) d y\right\rangle
$$

(by an argument similar to [6, Th. 2])

$\rightarrow\langle f, \phi\rangle$ as $\eta \rightarrow 0+$, since one can show that 
$\frac{1}{\pi} \int_{-\infty}^{\infty} \frac{\eta}{(t-y)^{2}+\eta^{2}} \phi(y) d y \rightarrow \phi(t)$ as $\eta \rightarrow 0+$, in the topology of $H_{\alpha}$.

IV. Discussion. Let $\Omega$ be the upper half-plane. If $f \in L^{P}(-\infty, \infty)$, we consider the Dirichlet problem:

$$
\begin{aligned}
\nabla^{2} u & =0 \text { in } \Omega \\
u(x, 0) & =f(x) .
\end{aligned}
$$

The solution to this is the Poisson Integral

$$
u(x, \eta)=\frac{\eta}{\pi} \int_{-\infty}^{\infty} \frac{f(t) d t}{(t-x)^{2}+\eta^{2}},
$$

whose harmonic conjugate is

$$
v(x, \eta)=-\frac{1}{\pi} \int_{-\infty}^{\infty} \frac{(t-x) f(t) d t}{(t-x)^{2}+\eta^{2}}
$$

Taking the limit of this as $\eta \rightarrow 0$ (in the $L^{P}$-norm) gives a function $g=-(1 / \pi) H f$. The functions $f$ and $g$ satisfy Hilbert's reciprocity relation $g=-(1 / \pi) H f: f=-\left(1 / \pi^{2}\right) H g$. The Hilbert transform of $f$, then, (ignoring a constant multiple of $-1 / \pi$ ) represents the boundary values of the harmonic conjugate of the solution to the Dirichlet problem for $f$. All this is well-known [9] for $L^{P}$-functions. In attempting to extend this theory to distributions it is natural to define the Hilbert transform of a distribution $f \in H_{\alpha}^{\prime}$ as

$$
\lim _{\eta \rightarrow 0+}\left\langle f(t), \frac{t-x}{(t-x)^{2}+\eta^{2}}\right\rangle=\lim _{\eta \rightarrow 0+} P_{\eta} f(x)
$$

provided that this limit exists in some suitable sense. It turns out that the interpretation of the limit is important: for example, if $f$ is taken to be the $\delta$-function, we get $P_{\eta} \delta(x)=-x /\left(x^{2}+\eta^{2}\right)$. This has an almosteverywhere pointwise limit, the point function $-1 / x$. It also has, in the weak distributional sense, the limit $-P V(1 / x)$, which is a distribution with values $\langle P V(1 / x), \phi\rangle=P \int_{-\infty}^{\infty}(1 / x) \phi(x) d x$ defined on the subspace of $H_{1}$ consisting of those functions $\phi$ such that $|\phi(x)|=O(1 /|x|)$ as $|x| \rightarrow \infty$.

The inversion properties of these two interpretations are quite different. In the latter case, we have the inversion formula $\delta=-\left(1 / \pi^{2}\right) H H \delta$, as can be seen by direct computation: $-\left(1 / \pi^{2}\right) P_{\eta} H \delta=-\left(1 / \pi^{2}\right) P_{\eta}(-P V(1 / x))=$ $(1 / \pi) \eta /\left(x^{2}+\eta^{2}\right)$, which is the Poisson Integral of $\delta$ (i.e. $1 / \pi\left\langle\delta(t), \eta /\left((t-x)^{2}+\right.\right.$ $\left.\left.\eta^{2}\right)\right\rangle$ ), and which converges to $\delta$ as $\eta \rightarrow 0+$, in the weak distributional sense. On the other hand, if we take the first interpretation (which, in this context, is admittedly somewhat unnatural), we find that the in- 
version formula fails. In particular, $H \delta(x)=-1 / x$, and if $\phi \in \mathscr{D}$, with support in $[a, b]$, then

$$
\begin{aligned}
\left\langle P \int_{-N}^{N} \frac{d x}{x(x-y)}, \phi(y)\right\rangle & =\left\langle\frac{1}{y} \ln \left|\frac{N-y}{N+y}\right|, \phi(y)\right\rangle=\int_{a}^{b} \frac{1}{y} \ln \left|\frac{N-y}{N+y}\right| \phi(y) d y \\
& =\int_{a / N}^{b / N} \frac{1}{z} \ln \left|\frac{1-z}{1+z}\right| \phi(N z) d z \rightarrow 0 \quad \text { as } \quad N \rightarrow \infty .
\end{aligned}
$$

Thus, interpreting everything in a "pointwise" sense leads to the result that the Hilbert transform of $\delta$ inverts not to $\delta$ but to the zero distribution (we note in passing that the use of $P_{\eta}$ facilitates computation of $H(P V(1 / x))$, since a "direct" approach, as the counterexample shows, gives the wrong value).

We can put the results of the paper into operator form as follows. Theorem 4 says that $\lim _{\eta \rightarrow 0+} H P_{\eta} f=f$, for all $f \in H_{\alpha}^{\prime}$. The " $H$ " in this formula is the "pointwise" Hilbert transform applied to $P_{\eta} f(x)$ as a function of $x$. If one continues to interpret $H$ in this sense, one cannot move the limit inside the integral in (23), as the above counterexample shows. But if $H$ is as in (24), with weak convergence, then the formula $-\left(1 / \pi^{2}\right) H H f=f$ holds if $f=\delta$ or if $f=P V(1 / x)$. Whether it holds in general is something we intend to investigate in a later paper, along with the exact conditions under which $\lim _{\eta \rightarrow 0} P_{\eta} f$ exists.

\section{REFERENCES}

[1] R. W. B. Best, Fouries and Hilbert transforms of generalized functions of one real variable, Associate Euratom-Fom, Fom-Institute voor plasma-Fysica, Rijnhuizen Jutphaas-Nederland, Rijnhuizen Report 66-130.

[2] E. J. Beltrami and M. R. Wohlers, Distributional boundary value theorems and Hilbert transform, Arch. Rational Mech. Anal., Vol. 18, (1965), 304-309.

[3] F. G. Tricomi, Integral Equations, Interscience, 1957.

[4] Marion Orton, Hilbert transforms, Plemelj relations and Fourier transforms of distributions, Siam J. Math. Anal., Vol. 4, November 1973, 656-667.

[5] J. N. PANDEY, On the Stieltjes transform of generalized functions, Proc. Camb. Phil. Soc. 71 (1972), 85-95.

[6] J. N. Pandey and A. H. Zemanian, Complex inversion for the generalized convolution transformation, Pacific J. Math., Vol. 25, No. 1, 147-157.

[7] J. N. PANDey and Edward Hughes, An approximate inversion formula for the Hilbert transform of generalized functions, Carleton Mathematics Series \#104, Carleton University.

[8] L. Schwartz, Theorie des distributions, Vol. I and II, Hermann, Paris, 1957 and 1959.

[9] E. C. Titchmarsh, Theory of Fourier integrals, Clarendon Press, 1948.

[10] A. H. Zemanian, Distribution theory and transform analysis, McGraw-Hill Book Company, 1965. 
[11] A. H. Zemanian, Generalized Integral Transformations, Interscience Publishers, 1968. DePARTMENT OF MATHEMATics

Carleton University

Ottawa, Canada 
